\title{
A CLINICAL AND RADIOLOGICAL PROFILE OF PATIENTS OF INTERSTITIAL LUNG DISEASES, ATTENDING THE CHEST HOSPITAL OF MEDICAL COLLEGE FROM NORTH INDIA
}

\author{
Anand Kumar', Sanjay Kumar Verma², Roshan Lal ${ }^{3}$, Avdhesh Kumar', Sudhir Chaudhri ${ }^{5}$, Rajendra Prasad6, Surya Kant ${ }^{7}$ \\ ${ }^{1}$ Assistant Professor, Department of Tuberculosis and Respiratory Diseases, GSVM Medical College, Kanpur. \\ ${ }^{2}$ Assistant Professor, Department of Tuberculosis and Respiratory Diseases, Government Medical College, Kannauj. \\ 3Junior Resident, Department of Tuberculosis and Respiratory Diseases, GSVM Medical College, Kanpur. \\ ${ }_{4}^{4}$ Assistant Professor, Department of Tuberculosis and Respiratory Diseases, GSVM Medical College, Kanpur. \\ ${ }^{5}$ Professor, Department of Tuberculosis and Respiratory Diseases, GSVM Medical College, Kanpur. \\ ${ }^{6}$ Professor and HOD, Department of Pulmonary Medicine, Era's Lucknow Medical College, Lucknow, India. \\ 7 Professor and HOD, Department of Pulmonary Medicine, King George's Medical University, UP, Lucknow, India.
}

\section{ABSTRACT}

\section{OBJECTIVE}

To know the clinical and radiological profile of patients of Interstitial Lung Diseases attending the Chest Hospital of Medical College from North India from February 2014 to December 2015.

\section{MATERIAL AND METHODS}

The study was conducted in Department of Tuberculosis and Respiratory Diseases, G.S.V.M. Medical College, Kanpur (India). The patients were subjected to complete clinical history (Including work environment and occupational history), physical examination, various haematological investigations, spirometry and high resolution computerized tomography thorax.

\section{RESULTS}

In present study a total of 80 patients with interstitial lung disease were analysed. There were 36 males and 44 females (mean age was $54.4 \pm 1.8$ years). Of 80 patients of interstitial lung diseases examined, Idiopathic Pulmonary Fibrosis (IPF) pattern was present in maximum number (38.8\%) followed by sarcoidosis (17.5\%), Hypersensitivity pneumonitis (15\%) Non-specific interstitial pneumonias (10\%), Connective Tissue Disorder (C.T.D.) associated ILD (7.5\%) and Cryptogenic Organizing Pneumonias (C.O.P.) in $5 \%$ of cases.

\section{CONCLUSION}

IPF was the most common subgroup among ILDs.

\section{KEYWORDS}

Interstitial Lung Diseases, Idiopathic Pulmonary Fibrosis, Sarcoidosis.

HOW TO CITE THIS ARTICLE: Kumar A, Verma SK, Lal R, et al. A clinical and radiological profile of patients of interstitial lung diseases, attending the chest hospital of medical college from North India. J. Evolution Med. Dent. Sci. 2016;5(41):2547-2552, DOI: $10.14260 /$ jemds/2016/595

\section{INTRODUCTION}

The term Diffuse Parenchymal Lung Disease (DPLD) often used synonymously with Interstitial Lung Disease (ILD), refers to diseases that cause inflammation of the pulmonary interstitium. About 200 disorders have been implicated in the causation of DPLD. ${ }^{1}$ The ILDs include diseases of unknown aetiology such as those secondary to drugs, collagen vascular disease, granulomatous conditions like sarcoidosis and other forms of ILDs like lymphangioleiomyomatosis or histiocytosis $\mathrm{X}$. Diagnosis can be made by the combination of clinical and roentgenographic features and pulmonary function tests. Histopathological confirmation of the diagnosis is not required in most of the cases. There has been a resurgence of interest in the study of these disorders chiefly on account of the availability of less invasive methods.

Financial or Other, Competing Interest: None.

Submission 05-04-2016, Peer Review 30-04-2016,

Acceptance 07-05-2016, Published 23-05-2016.

Corresponding Author:

Dr. Sudhir Chaudhri,

Professor,

Department of Tuberculosis and Respiratory Diseases,

GSVM Medical College, Kanpur.

E-mail: sudhirchaudhri@gmail.com.

DOI: $10.14260 /$ jemds $/ 2016 / 595$
Development of high resolution computed tomography and availability of video-assisted thoracoscopic lung biopsy has added to our diagnostic strategies. ${ }^{2}$

The present study was, therefore, planned to analyse the spectrum of ILD encountered in a tertiary referral centre and determine the clinical profile of the disease.

\section{MATERIAL AND METHODS}

The study was conducted in Department of Tuberculosis and Respiratory Diseases (Dr. M L Chest Hospital), G.S.V.M. Medical College, Kanpur (India) from February 2014 to December 2015. The patients were subjected to complete clinical history (Including work environment and occupational history) and complete physical examination.

Complete blood count, liver function test, kidney function test, serum electrolyte, fasting and post prandial blood sugar, urinalysis, sputum, chest X-ray - PA view, spirometry, diffusion study (If required), 2D Echo, High Resolution Computerized Tomography Thorax, 6 minute walk test. Other tests as per requirement would be done. Oxygen saturation and baseline Arterial Blood Gas analysis was done.

\section{Inclusion Criteria}

Patients Presenting with Following

- Respiratory symptoms such as shortness of breath and cough. 
- Bilateral abnormality in Chest X-ray/High Resolution Computerized Tomography scan thorax.

\section{Exclusion Criteria}

- Any infectious or malignant disease.

\section{RESULT}

In present study, a total of 80 patients with interstitial lung disease were analysed. There were 36 males and 44 females (Mean age was $54.4 \pm 1.8$ years). The female patients presented at an earlier age ( $54.4 \pm 1.8$ years) as compared to male patients (58.3 \pm 1.5 years); 37 patients were from rural background $(46.3 \%)$ and 43 were from urban area (53.8\%). Mean B.M.I. of ILD patients was $21.58 \pm 5.0 \mathrm{~kg} / \mathrm{m}^{2}$. Of 80 patients of interstitial lung diseases examined, Idiopathic Pulmonary Fibrosis (IPF) pattern was present in maximum number (38.8\%) followed by sarcoidosis $(17.5 \%)$, hypersensitivity pneumonitis $(15 \%)$, non-specific interstitial pneumonias (10\%), Connective Tissue Disorder (C.T.D.), associated ILD (7.5\%) and Cryptogenic Organizing Pneumonias (C.O.P.) in 5\% of cases (Table 1).

In ILD patients $95.9 \%$ presented with cough, among which $39.7 \%$ had expectoration, while $56.2 \%$ patients had dry cough and $4.1 \%$ of patients did not have cough as a presenting symptom ( $p=0.0624)$. Breathlessness was most consistent symptom present in all patients. Chest pain was present in $38.8 \%$ of ILD patients, most commonly associated with NSIP (75\%) and sarcoidosis (42.9\%).

Weight loss, difficulty in swallowing, skin changes and pedal oedema were found in $36.3 \%, 5 \%, 6.3 \%$ and $16.3 \%$ patients respectively. Bibasilar crepitation was the most consistent sign (95.5\%), clubbing and pallor were present in $61.1 \%$ and $58.9 \%$ of ILD patients. Joint pain was another symptom and was present in $27.8 \%$ cases in our study.

In this study, the percentage of non-smoker, ex-smoker and current smoker were $71.3 \%, 17.5 \%$ and $11.3 \%$ respectively. The disease wise details are given in Table No. 2 .

In present study, the details of exposure to various noxious substance and environmental factors in relation to various ILDs are given in Table 2.

The presence of co-morbidity was not unusual with DPLD; but in present study, most frequently present co-morbidity was hypertension and diabetes seen in $16.4 \%$ and $12.3 \%$ cases. In this study, gastroesophageal reflux as a symptom was seen in $17.8 \%$ cases; highest amongst IPF (24.1\%). In present study $31.5 \%$ cases of ILD, misdiagnosed as pulmonary tuberculosis and had a history of anti-tubercular treatment. This might be due to radiological similarities between ILD and pulmonary tuberculosis and a lack of awareness and paucity of diagnostic facilities in remote areas. (The description of other medical histories are summarized in Table 3).

$59(80.8 \%)$ patients with ILD were able to perform 6 minute walk test (6 MWT) and Mean expected 6 minute walk test distance was $658 \pm 94$ meters, while performed distance was $278 \pm 131$ meters (43.5\%). (The details are summarized in Table No. 2).

Spirometry was performed by 55 patients only and in Spirometry most common abnormality among ILD patients was restrictive pattern in $78.2 \%$, was normal in $10.9 \%$ cases.

Majority of patients shows reticular or reticulonodular patterns on chest X-ray and on HRCT of chest various radiological abnormality were Interseptal Thickening (72.6\%), Honeycombing (54.8\%), Bronchiectasis (60.2\%), GGOs (61.6\%), Reticulation (65.7\%), Cyst (5.5\%), Nodules (20.5\%), Air trapping (26.6\%), Intrathoracic lymph nodes (37.0\%), Pleural effusion (1.4\%), Pleural Thickening (15.1\%) and Consolidation (8.21\%). (The details are summarized in Table No. 2).

\begin{tabular}{|c|c|c|}
\hline Disease & $\begin{array}{c}\text { No. of } \\
\text { Patients }\end{array}$ & Percentage \\
\hline $\begin{array}{l}\text { Idiopathic pulmonary } \\
\text { fibrosis (I.P.F.) }\end{array}$ & 31 & $38.8 \%$ \\
\hline Sarcoidosis & 14 & $17.5 \%$ \\
\hline $\begin{array}{l}\text { Hypersensitivity } \\
\text { pneumonitis (H.P.) }\end{array}$ & 12 & $15 \%$ \\
\hline $\begin{array}{l}\text { Non-Specific Interstitial } \\
\text { pneumonias (N.S.I.P.) }\end{array}$ & 08 & $10 \%$ \\
\hline $\begin{array}{l}\text { Cryptogenic Organizing } \\
\text { Pneumonias (C.O.P.) }\end{array}$ & 04 & $5 \%$ \\
\hline $\begin{array}{c}\text { Connective Tissue Disorder } \\
\text { (C.T.D.) Associated ILD }\end{array}$ & 06 & $7.5 \%$ \\
\hline Others & 05 & $6.3 \%$ \\
\hline Total & 80 & $100 \%$ \\
\hline \multicolumn{3}{|c|}{$\begin{array}{l}\text { Table 1: Various Diagnoses Found among Patients of } \\
\text { Interstitial Lung Diseases }\end{array}$} \\
\hline
\end{tabular}

\begin{tabular}{|c|c|c|c|c|c|c|c|c|c|c|}
\hline $\begin{array}{l}\text { Sl. } \\
\text { No. }\end{array}$ & \multicolumn{2}{|c|}{ Parameter } & $\begin{array}{c}\text { IPF } \\
(n=31)\end{array}$ & $\begin{array}{c}\text { Sarcoidosis } \\
\quad(n=14)\end{array}$ & $\underset{(n=12)}{H P}$ & $\begin{array}{l}\text { NSIP } \\
(n=8)\end{array}$ & $\begin{array}{c}\text { COP } \\
(n=4)\end{array}$ & $\begin{array}{l}\text { CTD- } \\
\text { ILD }\end{array}$ & $\begin{array}{c}\text { Others } \\
(n=5)\end{array}$ & $\begin{array}{c}\text { Total } \\
(n=80)\end{array}$ \\
\hline \multirow{2}{*}{1} & \multirow{2}{*}{ Mean age } & Male & $\begin{array}{c}62.8 \\
\pm \\
1.7\end{array}$ & $\begin{array}{c}54.0 \\
\pm \\
4.2\end{array}$ & 45.0 & 70.0 & 52.5 & 37.0 & $\begin{array}{c}45.2 \\
\pm \\
22.2\end{array}$ & $\begin{array}{c}58.3 \\
\pm \\
1.5\end{array}$ \\
\hline & & Female & $\begin{array}{c}61.7 \\
\pm \\
1.7\end{array}$ & $\begin{array}{c}41.9 \\
\pm \\
4.2\end{array}$ & $\begin{array}{c}63.1 \\
\pm \\
8.0\end{array}$ & $\begin{array}{c}47.5 \\
\pm \\
1.7\end{array}$ & $\begin{array}{c}53.0 \\
\pm \\
31.8\end{array}$ & $\begin{array}{c}39.7 \\
\pm \\
11.8\end{array}$ & 54.0 & $\begin{array}{c}54.4 \\
\pm \\
1.8\end{array}$ \\
\hline \multirow{5}{*}{2} & \multirow{5}{*}{$\begin{array}{c}\text { Age } \\
\text { Distribution } \\
\text { (Years) }\end{array}$} & $>30$ & 0 & $\begin{array}{c}4 \\
(28.6)\end{array}$ & 0 & $\begin{array}{c}3 \\
(37.5)\end{array}$ & 0 & $\begin{array}{c}1 \\
(16.7)\end{array}$ & $\begin{array}{c}1 \\
(20)\end{array}$ & $\begin{array}{c}9 \\
(11.3)\end{array}$ \\
\hline & & 30-39 & $\begin{array}{c}2 \\
(6.5) \\
\end{array}$ & $\begin{array}{c}1 \\
(7.1)\end{array}$ & 0 & 0 & $\begin{array}{c}2 \\
(50) \\
\end{array}$ & $\begin{array}{c}2 \\
(33.3) \\
\end{array}$ & 0 & $\begin{array}{c}7 \\
(8.8)\end{array}$ \\
\hline & & $40-49$ & $\begin{array}{c}5 \\
(16.1)\end{array}$ & $\begin{array}{c}4 \\
(28.6)\end{array}$ & $\begin{array}{c}3 \\
(25)\end{array}$ & 0 & 0 & $\begin{array}{c}2 \\
(33.3)\end{array}$ & $\begin{array}{c}2 \\
(40)\end{array}$ & $\begin{array}{c}16 \\
(20)\end{array}$ \\
\hline & & $50-59$ & $\begin{array}{c}6 \\
(19.4)\end{array}$ & $\begin{array}{c}4 \\
(28.6)\end{array}$ & $\begin{array}{c}1 \\
(8.3)\end{array}$ & $\begin{array}{c}2 \\
(25)\end{array}$ & $\begin{array}{c}1 \\
(25)\end{array}$ & $\begin{array}{c}1 \\
(16.7)\end{array}$ & $\begin{array}{c}1 \\
(20)\end{array}$ & $\begin{array}{c}16 \\
(20)\end{array}$ \\
\hline & & $60-69$ & $\begin{array}{c}5 \\
(16.1)\end{array}$ & $\begin{array}{c}1 \\
(7.1)\end{array}$ & $\begin{array}{c}6 \\
(50)\end{array}$ & $\begin{array}{c}2 \\
(25)\end{array}$ & 0 & 0 & 0 & $\begin{array}{c}14 \\
(22.5)\end{array}$ \\
\hline
\end{tabular}




\begin{tabular}{|c|c|c|c|c|c|c|c|c|c|c|}
\hline & & $>70$ & $\begin{array}{c}13 \\
(41.9)\end{array}$ & 0 & $\begin{array}{c}2 \\
(16.7)\end{array}$ & $\begin{array}{c}1 \\
(12.5)\end{array}$ & $\begin{array}{c}1 \\
(25) \\
\end{array}$ & 0 & $\begin{array}{c}1 \\
(20)\end{array}$ & $\begin{array}{c}18 \\
(24.6) \\
\end{array}$ \\
\hline \multirow{2}{*}{3} & \multirow{2}{*}{ Gender } & Male & $\begin{array}{c}22 \\
(71)\end{array}$ & $\begin{array}{c}2 \\
(14.3) \\
\end{array}$ & $\begin{array}{c}2 \\
(16.7) \\
\end{array}$ & $\begin{array}{c}2 \\
(25) \\
\end{array}$ & $\begin{array}{c}2 \\
(50) \\
\end{array}$ & $\begin{array}{c}2 \\
(33.3) \\
\end{array}$ & $\begin{array}{c}4 \\
(80) \\
\end{array}$ & $\begin{array}{c}36 \\
(45) \\
\end{array}$ \\
\hline & & Female & $\begin{array}{c}9 \\
(29)\end{array}$ & $\begin{array}{c}12 \\
(85.7)\end{array}$ & $\begin{array}{c}10 \\
(83.3)\end{array}$ & $\begin{array}{c}6 \\
(75)\end{array}$ & $\begin{array}{c}2 \\
(50)\end{array}$ & $\begin{array}{c}4 \\
(66.7)\end{array}$ & $\begin{array}{c}1 \\
(20)\end{array}$ & $\begin{array}{c}44 \\
(55)\end{array}$ \\
\hline \multirow{2}{*}{4} & \multirow{2}{*}{ Residence } & Rural & $\begin{array}{c}18 \\
(58.1)\end{array}$ & $\begin{array}{c}4 \\
(28.6)\end{array}$ & $\begin{array}{c}7 \\
(58.3)\end{array}$ & $\begin{array}{c}4 \\
(50)\end{array}$ & $\begin{array}{c}1 \\
(25)\end{array}$ & $\begin{array}{c}2 \\
(50)\end{array}$ & $\begin{array}{c}1 \\
(20)\end{array}$ & $\begin{array}{c}37 \\
(46.3)\end{array}$ \\
\hline & & Urban & $\begin{array}{c}13 \\
(41.9) \\
\end{array}$ & $\begin{array}{c}10 \\
(71.4)\end{array}$ & $\begin{array}{c}5 \\
(41.7) \\
\end{array}$ & $\begin{array}{c}4 \\
(50) \\
\end{array}$ & $\begin{array}{c}3 \\
(75) \\
\end{array}$ & $\begin{array}{c}4 \\
(50) \\
\end{array}$ & $4(80)$ & $43(53.8)$ \\
\hline \multirow{4}{*}{5} & \multirow{4}{*}{$\begin{array}{l}\text { Exposure to } \\
\text { various } \\
\text { noxious \& toxic } \\
\text { substance }\end{array}$} & $\begin{array}{l}\text { Cooking on } \\
\text { fire wood/ } \\
\text { cow dung }\end{array}$ & $\begin{array}{c}26 \\
(83.9)\end{array}$ & $\begin{array}{c}6 \\
(42.9)\end{array}$ & $\begin{array}{c}9 \\
(75)\end{array}$ & $\begin{array}{c}6 \\
(75)\end{array}$ & $\begin{array}{c}2 \\
(50)\end{array}$ & $\begin{array}{c}2 \\
(33.3)\end{array}$ & $\begin{array}{c}2 \\
(40)\end{array}$ & $\begin{array}{c}53 \\
(66.3)\end{array}$ \\
\hline & & Kerosene & $\begin{array}{c}12 \\
(38.7)\end{array}$ & $\begin{array}{c}2 \\
(14.3)\end{array}$ & $\begin{array}{c}4 \\
(33.3)\end{array}$ & $\begin{array}{c}3 \\
(37.5)\end{array}$ & $\begin{array}{c}2 \\
(50)\end{array}$ & $\begin{array}{c}2 \\
(33.3)\end{array}$ & $\begin{array}{c}1 \\
(20)\end{array}$ & $\begin{array}{c}26 \\
(32.5)\end{array}$ \\
\hline & & Coal & $\begin{array}{c}6 \\
(19.4)\end{array}$ & $\begin{array}{c}1 \\
(7.1)\end{array}$ & $\begin{array}{c}2 \\
(16.7)\end{array}$ & 0 & 0 & 0 & 0 & $\begin{array}{c}09 \\
(11.3)\end{array}$ \\
\hline & & LPG & $\begin{array}{c}17 \\
(54.8)\end{array}$ & $\begin{array}{c}12 \\
(85.7)\end{array}$ & $\begin{array}{c}8 \\
(66.7)\end{array}$ & $\begin{array}{c}4 \\
(50)\end{array}$ & $\begin{array}{c}3 \\
(75)\end{array}$ & $\begin{array}{c}3 \\
(50)\end{array}$ & $\begin{array}{c}4 \\
(80)\end{array}$ & $\begin{array}{c}51 \\
(63.8)\end{array}$ \\
\hline \multirow{5}{*}{6} & \multirow{5}{*}{$\begin{array}{l}\text { Exposure to } \\
\text { various } \\
\text { environmental } \\
\text { factors }\end{array}$} & Dust & $\begin{array}{c}13 \\
(41.9)\end{array}$ & $\begin{array}{c}6 \\
(42.9)\end{array}$ & $\begin{array}{c}2 \\
(16.7)\end{array}$ & $\begin{array}{c}1 \\
(12.5)\end{array}$ & $\begin{array}{c}2 \\
(50)\end{array}$ & $\begin{array}{c}1 \\
(16.7)\end{array}$ & $\begin{array}{c}2 \\
(40)\end{array}$ & $\begin{array}{c}27 \\
(33.8)\end{array}$ \\
\hline & & Mold & $\begin{array}{c}4 \\
(12.9) \\
\end{array}$ & $\begin{array}{c}2 \\
(14.9) \\
\end{array}$ & $\begin{array}{c}2 \\
(16.7) \\
\end{array}$ & 0 & 0 & $\begin{array}{c}2 \\
(50) \\
\end{array}$ & $\begin{array}{c}1 \\
(20) \\
\end{array}$ & $\begin{array}{c}11 \\
(13.8)\end{array}$ \\
\hline & & $\mathrm{AC}$ & $\begin{array}{c}3 \\
(9.7) \\
\end{array}$ & $\begin{array}{c}5 \\
(35.7) \\
\end{array}$ & $\begin{array}{c}3 \\
(25) \\
\end{array}$ & $\begin{array}{c}2 \\
(25) \\
\end{array}$ & 0 & 0 & $\begin{array}{c}2 \\
(40) \\
\end{array}$ & $\begin{array}{c}15 \\
(18.8)\end{array}$ \\
\hline & & Cooler & $\begin{array}{c}17 \\
(54.8)\end{array}$ & $\begin{array}{c}13 \\
(92.9)\end{array}$ & $\begin{array}{c}11 \\
(91.7)\end{array}$ & $\begin{array}{c}5 \\
(62.5)\end{array}$ & $\begin{array}{c}3 \\
(75)\end{array}$ & 0 & $\begin{array}{c}4 \\
(80)\end{array}$ & $\begin{array}{c}53 \\
(66.3)\end{array}$ \\
\hline & & Birds/pets & $\begin{array}{c}2 \\
(6.5) \\
\end{array}$ & $\begin{array}{c}1 \\
(7.1)\end{array}$ & $\begin{array}{c}3 \\
(25) \\
\end{array}$ & 0 & 0 & $\begin{array}{c}1 \\
(16.7) \\
\end{array}$ & 0 & $\begin{array}{c}7 \\
(8.8)\end{array}$ \\
\hline \multirow{3}{*}{7} & \multirow{3}{*}{$\begin{array}{l}\text { Tobacco } \\
\text { smoker }\end{array}$} & $\begin{array}{c}\text { Non- } \\
\text { smoker }\end{array}$ & $\begin{array}{c}18 \\
(58.1 \%) \\
\end{array}$ & $\begin{array}{c}11 \\
(78.6 \%) \\
\end{array}$ & $\begin{array}{c}10 \\
(83.3 \%) \\
\end{array}$ & $\begin{array}{c}7 \\
(87.5 \%) \\
\end{array}$ & $\begin{array}{c}3 \\
(75 \%) \\
\end{array}$ & $\begin{array}{c}5 \\
(83.3 \%) \\
\end{array}$ & $\begin{array}{c}3 \\
(60 \%) \\
\end{array}$ & $\begin{array}{c}57 \\
(71.3)\end{array}$ \\
\hline & & Ex-smoker & $\begin{array}{c}7 \\
(22.6 \%) \\
\end{array}$ & $\begin{array}{c}2 \\
(14.3 \%) \\
\end{array}$ & $\begin{array}{c}2 \\
(16.7 \%) \\
\end{array}$ & $\begin{array}{c}1 \\
(12.5 \%) \\
\end{array}$ & $\begin{array}{c}1 \\
(25 \%) \\
\end{array}$ & 0 & $\begin{array}{c}1 \\
(20 \%) \\
\end{array}$ & $\begin{array}{c}14 \\
(17.5)\end{array}$ \\
\hline & & $\begin{array}{l}\text { Current } \\
\text { smoker }\end{array}$ & $\begin{array}{c}6 \\
(19.4 \%) \\
\end{array}$ & $\begin{array}{c}1 \\
(7.1 \%) \\
\end{array}$ & 0 & 0 & 0 & $\begin{array}{c}1 \\
(16.7 \%) \\
\end{array}$ & $\begin{array}{c}1 \\
(20 \%)\end{array}$ & $\begin{array}{c}9 \\
(11.3)\end{array}$ \\
\hline \multirow[t]{2}{*}{8} & \multirow[t]{2}{*}{6 MWD } & $\begin{array}{l}\text { Expected } \\
\text { walk } \\
\text { distance } \\
\end{array}$ & $\begin{array}{l}640 \\
\pm 94\end{array}$ & $\begin{array}{l}686 \\
\pm 78\end{array}$ & $\begin{array}{l}596 \\
\pm 78\end{array}$ & $\begin{array}{l}709 \\
\pm 82\end{array}$ & $\begin{array}{c}751 \\
\pm 152\end{array}$ & $\begin{array}{l}741 \\
\pm 90\end{array}$ & $\begin{array}{l}691 \\
\pm 84\end{array}$ & $\begin{array}{l}658 \\
\pm 94\end{array}$ \\
\hline & & $\begin{array}{l}\text { Actual walk } \\
\text { distance }\end{array}$ & $\begin{array}{c}269 \\
\pm 125 \\
\end{array}$ & $\begin{array}{c}287 \\
\pm 131 \\
\end{array}$ & $\begin{array}{c}272 \\
\pm 146 \\
\end{array}$ & $\begin{array}{r}315 \\
\pm 180 \\
\end{array}$ & $\begin{array}{l}420 \\
\pm 42 \\
\end{array}$ & $\begin{array}{r}326 \\
\pm 126 \\
\end{array}$ & $\begin{array}{l}182 \\
\pm 17 \\
\end{array}$ & $\begin{array}{c}278 \\
\pm 131 \\
\end{array}$ \\
\hline 9 & \multicolumn{2}{|c|}{ Pulmonary hypertension } & $06.8 \%$ & $7.7 \%$ & $27.2 \%$ & $14.2 \%$ & 00 & $20 \%$ & 00 & $12.3 \%$ \\
\hline \multirow{13}{*}{10} & \multirow{13}{*}{$\begin{array}{c}\text { Various } \\
\text { radiological } \\
\text { findings }\end{array}$} & $\begin{array}{l}\text { Interseptal } \\
\text { thickening }\end{array}$ & $100 \%$ & $30.7 \%$ & $63.6 \%$ & $85.7 \%$ & $33.3 \%$ & $80 \%$ & $60 \%$ & $72.6 \%$ \\
\hline & & $\begin{array}{c}\text { Honey } \\
\text { combing }\end{array}$ & $86.2 \%$ & $15.4 \%$ & $54.5 \%$ & $14.2 \%$ & $66.6 \%$ & $60 \%$ & $20 \%$ & $54.8 \%$ \\
\hline & & Bronchiectasis & $86.2 \%$ & $30.7 \%$ & $54.5 \%$ & $28.4 \%$ & $66.6 \%$ & $60 \%$ & $40 \%$ & $60.2 \%$ \\
\hline & & GGOs & $51.2 \%$ & $53.8 \%$ & $63.3 \%$ & $100 \%$ & $66.6 \%$ & $80 \%$ & $60 \%$ & $61.6 \%$ \\
\hline & & Reticulations & $96.5 \%$ & $23.1 \%$ & $63.3 \%$ & $56.8 \%$ & $33.3 \%$ & $80 \%$ & $20 \%$ & $65.7 \%$ \\
\hline & & Cyst & $3.4 \%$ & 00 & $27.2 \%$ & 00 & 00 & 00 & 00 & $5.5 \%$ \\
\hline & & Nodules & $17.2 \%$ & $30.8 \%$ & $36.3 \%$ & 00 & 00 & $20 \%$ & $20 \%$ & $20.5 \%$ \\
\hline & & Air Trapping & $17.2 \%$ & $15.4 \%$ & $27.2 \%$ & $14.2 \%$ & 00 & $40 \%$ & $40 \%$ & $26.6 \%$ \\
\hline & & $\begin{array}{c}\text { Intrathoracic } \\
\text { LN } \\
\end{array}$ & $24.1 \%$ & $92.3 \%$ & $18.2 \%$ & $14.2 \%$ & $33.3 \%$ & $20 \%$ & $60 \%$ & $37.0 \%$ \\
\hline & & $\begin{array}{c}\text { Pleural } \\
\text { effusion }\end{array}$ & 00 & 00 & 00 & 00 & 00 & 00 & $20 \%$ & $01.4 \%$ \\
\hline & & $\begin{array}{c}\text { Pleural } \\
\text { thickening }\end{array}$ & $13.8 \%$ & $7.7 \%$ & $18.2 \%$ & $28.4 \%$ & 00 & $20 \%$ & $20 \%$ & $15.1 \%$ \\
\hline & & Consolidation & 00 & $7.7 \%$ & 0 & $14.2 \%$ & $66.6 \%$ & $20 \%$ & 00 & $8.21 \%$ \\
\hline & & UIP Pattern & $93.2 \%$ & $7.7 \%$ & $36.3 \%$ & 00 & 00 & $60 \%$ & $20 \%$ & $49.3 \%$ \\
\hline
\end{tabular}




\begin{tabular}{|c|c|c|c|c|c|c|c|c|c|}
\hline \multirow{2}{*}{$\begin{array}{l}\text { Sl. } \\
\text { No. }\end{array}$} & \multirow{2}{*}{$\begin{array}{c}\text { Past Medical } \\
\text { H/O }\end{array}$} & \multicolumn{7}{|c|}{ Variety of ILD } & \multirow{2}{*}{$\begin{array}{c}\text { Total } \\
(n=80)\end{array}$} \\
\hline & & $\begin{array}{c}\text { IPF } \\
(n=31)\end{array}$ & $\begin{array}{c}\text { Sarcoidosis } \\
(n=14)\end{array}$ & $\begin{array}{c}\text { HP } \\
(n=12)\end{array}$ & $\begin{array}{c}\text { NSIP } \\
(n=8)\end{array}$ & $\begin{array}{c}\text { COP } \\
(n=4)\end{array}$ & $\begin{array}{c}\text { CTD-ILD } \\
(n=6)\end{array}$ & $\begin{array}{c}\text { Others } \\
(n=5)\end{array}$ & \\
\hline 1 & H/o ATT & $31.1 \%$ & $53.8 \%$ & $18.9 \%$ & $28.4 \%$ & $33.3 \%$ & 0 & $40 \%$ & $31.5 \%$ \\
\hline 2 & Hypertension & $6.9 \%$ & $23.1 \%$ & $27.2 \%$ & $42.8 \%$ & $33.3 \%$ & 0 & 0 & $16.4 \%$ \\
\hline 3 & Diabetes & $3.4 \%$ & $15.3 \%$ & $36.3 \%$ & $28.4 \%$ & 0 & 0 & 0 & $12.3 \%$ \\
\hline 4 & Thyroid disease & $3.4 \%$ & $15.3 \%$ & $18.8 \%$ & 0 & 0 & 0 & 0 & $6.8 \%$ \\
\hline 5 & Kidney disease & $3.4 \%$ & $7.7 \%$ & 0 & 0 & 0 & 0 & 0 & $2.9 \%$ \\
\hline 6 & Pleural effusion & $3.4 \%$ & $7.7 \%$ & $9.1 \%$ & 0 & 0 & $20 \%$ & $40 \%$ & $8.21 \%$ \\
\hline 7 & G.E.R.D. & $24.1 \%$ & $15.4 \%$ & $9.1 \%$ & $14.1 \%$ & 0 & $20 \%$ & $20 \%$ & $17.8 \%$ \\
\hline
\end{tabular}

\section{DISCUSSION}

This was a hospital based prospective, observational study of patients with Interstitial Lung Diseases (ILD), which included detailed clinical history (Including environmental, occupational and medical history), physical examination, radiological and other relevant investigations.

80 patients of ILD were included in the study and in this study there were $64(80 \%)$ patients $>40$ years of age, which is comparable to a study done in Kuwait. ${ }^{3}$ A study from India found that peak incidence was between 40-49 years' age group and then 30-39 years' age group. ${ }^{4}$ Jindal et al showed that peak incidence was between 30 to 59 years. ${ }^{5}$ In another study done revealed the peak incidence was in 20-29 and 40-49 years' age group. 6

Mean age in present study was $54.4 \pm 1.8$ years $(58.3 \pm 1.5$ in males and $51.9 \pm 1.6$ years in females). The mean age of patients in various other studies varied from 43.5 to 52.4 years. ${ }^{4,7-10}$

In present study, ILD was seen more in females $44 / 80$ (55\%) than males 36/80 (45\%). Various authors reported variable findings on either sex predominance. Contrary to our study, Gagiya et al showed male preponderance (66.5\%). ${ }^{4}$ Another study from India reported that females were twice compared to male. ${ }^{7}$ In another study, male and female incidence was $42.4 \%$ and $57.4 \%$ respectively. ${ }^{5}$ A study from Lucknow, India, reported that the prevalence of DPLD among male and female were $66.5 \%$ and $33.5 \%$ respectively. ${ }^{6}$ Another study from India revealed the prevalence of diffuse fibrosing alveolitis among male and female were $53 \%$ and $47 \%$ respectively. ${ }^{11} \mathrm{~A}$ study from Trivandrum, India, found $48.6 \%$ males and $51.4 \%$ females among ILDs patients. ${ }^{9} \mathrm{D}$ B Coultas et al, studied to describe the prevalence and incidence of ILDs a population-based registry in Bernalillo County, New Mexico. In October 1988, the prevalence of ILDs was $20 \%$ higher in males $(80.9$ per 100,000$)$ than in females $(67.2$ per 100,000$)$. Similarly, the overall incidence of ILDs was slightly more common in males (31.5 per $100,000 /$ year) than females $(26.1$ per $100,000 /$ year). ${ }^{12}$

The interstitial lung diseases are diverse group of over 150 disorders. ${ }^{13}$ In our study, among the 80 cases Idiopathic Pulmonary Fibrosis (IPF) was present in maximum number of patients (38.8\%), followed by Sarcoidosis (17.5\%), Hypersensitivity pneumonitis (15\%), Non-specific interstitial pneumonias (10\%) and Connective tissue disorder associated with ILDs in $7.5 \%$ cases.

A study from Mexico also found that the most common disease diagnosed among both sexes were idiopathic pulmonary fibrosis accounting for $46.2 \%$ of all ILD diagnoses in males and $44.2 \%$ in females. ${ }^{12}$ Another study from Lucknow (India) reported IPF, Cryptogenic Organising Pneumonia
(COP), Non-Specific Interstitial Pneumonia (NSIP) and Sarcoidosis in 48.7\%, 20.5\%, $17.9 \%$ and 7\%, respectively. ${ }^{6}$

Tiyas Sen et al, observed idiopathic pulmonary fibrosis, sarcoidosis and ILDs secondary to connective tissue disorder in $43 \%, 22 \%$ and $19 \%$ respectively. ${ }^{7}$ S Kundu et al concluded that Idiopathic Pulmonary Fibrosis (IPF) was the most common variety in $38.04 \%$ followed by CTD in $31.5 \%$, hypersensitivity pneumonitis (10.9\%), sarcoidosis (5.4\%) and silicosis (5.4\%) being the common causes of ILDs. ${ }^{14}$ Rajkumar et al reported that sarcoidosis (37.3\%) was found to be the most common subgroup followed by IPF (27.6\%) and NSIP $(25.6 \%)$ among study group with various ILDs. ${ }^{10}$ Another study from Trivandrum (India) found Idiopathic pulmonary fibrosis (39\%), CTD associated ILD (24\%), Hypersensitivity Pneumonitis $17 \%$ and Sarcoidosis $13 \%$ in study group. ${ }^{9}$ Gagiya et al, found Idiopathic Pulmonary Fibrosis and Rheumatoid Arthritis in $33.3 \%$ and $13.2 \%$ respectively in various patients of ILDs. ${ }^{4}$ A study from Saudi Arabia reported that the most frequent disease among newly diagnosed ILDs were Connective Tissue Disease (CTD)-associated ILD (34.8\%) followed by Idiopathic Pulmonary Fibrosis (IPF) (23.3\%), Sarcoidosis (20\%), and Hypersensitivity Pneumonitis (6.3\%). ${ }^{3}$

In our study, IPF was more common in males (71\%) than females (29\%). Similar observations were made in the study conducted by Turner et al, where out of 220 IPF cases $66.8 \%$ were males and $32.2 \%$ were females. ${ }^{15}$ In the study done by Maheshwari et al, they found that there were 35 males and 41 females. ${ }^{8}$ In a study done by Subhash et al, amongst 33 patients who had IPF 17 were males. ${ }^{16}$ Our finding was also supported by Raghu et al, where more men were reported with IPF than women. ${ }^{17}$

In present study, two-thirds of the IPF cases present in patients over the age of 60 years with a mean age of 66 years at the time of diagnosis and mean age of presentation in IPF was $61.9 \pm 1.3$ years. In the study conducted by Turner et al, the mean age at presentation was 54 years \pm 12 SD years. ${ }^{15}$ In Maheshwari et al study, the mean age was $50.6 \pm 11.9$ SD years. ${ }^{8}$ In our study, there was no significant difference between the mean age of males $(62.8 \pm 1.7$ years) and females $(61.7 \pm 1.7$ years) amongst IPF cases.

In present study, gastroesophageal reflux as a symptom was seen in $17.8 \%$ cases; highest amongst IPF (24.1\%). A study conducted by Shafeeq $M$ et al reported the incidence of GERD to be in $20 \%$ of ILD cases. ${ }^{9}$ Another study done by Savarino E et al finds IPF patients had significantly higher $(\mathrm{p}<0.01)$ oesophageal acid exposure 9.25 (4.7-15.4\%) versus 3.3 (1.4$7.4 \%)$ versus $0.7(0.2-4.2 \%)$ as compared to non-IPF patients and healthy volunteers, respectively. ${ }^{18}$ 
Schwartz DA et al done a study in 73 confirmed cases of IPF and of them $62 \%$ were men and $70 \%$ were either former or current cigarette smokers.

They concluded that among patients with IPF, current cigarette smokers will tend to trap air (Higher RV and FRC) and that cigarette smoking appears to adversely alter gas exchange. 19

In this study, 28.8\% patients were smokers (Both current and ex-smokers) and majority of them were in IPF. A study done by Shafeeq MK et al reported smoking in 37\% patients in idiopathic pulmonary fibrosis. ${ }^{9}$

In our study, sarcoidosis was more commonly seen in females $(85.7 \%)$ and which is supported by most studies, which suggest a slightly higher disease rate for women. ${ }^{18}$ Benjamin et al studied and found that the female preponderance of sarcoidosis appears to be consistent across ethnic groups. ${ }^{20}$ Mean age of presentation of sarcoidosis in our study $43.7 \pm 3.6$ SD years. Similar observation was also made by Benjamin et al. ${ }^{21}$

Most common pattern in spirometry in ILD is restrictive. ${ }^{22}$ In our study, it was seen in $87.3 \%$ cases. While in other various studies, restrictive pattern ranged from $84.4 \%$ to $98 \% .4,6,9$

In present study, Pulmonary Hypertension by 2D Echocardiography was seen in $12.3 \%$ cases. While in other studies, it was ranged from $17-30 \% .{ }^{9-10,14}$ A study from India reported pulmonary hypertension in $38 \%$ patients with DPLD on the basis of clinical, radiological and electrocardiogram findings. ${ }^{5}$ A study from Denmark reported pulmonary hypertension in $14 \%$ with mixed types of ILD and who were screened for $\mathrm{PH}$ by echocardiography and concluded that $8 \%$ of them had mild PH (Mean PAP $>25 \mathrm{mmHg}$, but $<35 \mathrm{mmHg}$ ) and $6 \%$ of them had severe PH (Mean PAP $>35 \mathrm{mmHg}$ ). ${ }^{23-24}$ Hamada et al observed that the prevalence of $\mathrm{PH}$ in this patient population was as low as $8.1 \% .25$

Abeles AM et al reported that C-Reactive Protein was raised in $45.2 \%$ cases and was the most consistent finding followed by serum ANA positivity in $17.8 \%$ cases (Maximum in IPF: $27.6 \%$ ) and also found that Serum DsDNA was positive in $6.9 \%$ cases with IPF and $20 \%$ in CTD associated ILDs. ${ }^{26}$ Weinberg et al found ANA were positive in 10 of 34 of the patients screened and 2 patients with sarcoidosis had antibodies to dsDNA. ${ }^{27}$ Fischer et al reported that ANA and RF are poor screening tests because they have low specificity, particularly when present at low titer and can be seen in healthy individuals. ${ }^{28}$

In our study, interstitial septal thickening was most consistent radiological finding seen in $72.6 \%$ cases; other findings were reticulation (65.7\%), ground glass opacity (61.6\%), tractional bronchiectasis $(60.2 \%)$, honeycombing (54.8\%) mediastinal lymph node (37.0\%) and air trapping (26.6\%) cases. Other infrequent radiological finding was nodules (20\%), pleural thickening (8.21\%) and cyst in 5.5\% cases, which is most frequent in hypersensitivity pneumonitis. A study from India, studied HRCT Thorax of 70 DPLDs cases and they found Ground glass opacity $46 \%$, Septal thickening $69 \%$, Honeycombing 43\%, Nodules 16\% Mediastinal nodes $11.4 \%$ cases. ${ }^{9}$ Another one from India studied and found the overall patterns documented on HRCT $(n=289)$ were Interstitial fibrosis (49.9\%), Honeycombing (37.71\%), Ground glass opacities $(34.25 \%)$, Intrathoracic lymphadenopathy (20.76\%), Traction bronchiectasis (17.64\%) and Pleural fibrosis (5.8\%). Honeycombing was present in $4.62 \%$ cases of sarcoidosis, $31.08 \%$ cases of NSIP and in all IPF cases. ${ }^{10}$ Maheshwari et al studied HRCT thorax of 69 IPF cases and found all patients had features suggestive of diffuse interstitial fibrosis. Other abnormalities noted on HRCT chest included Honeycombing (88.4\%), Ground glass haziness (23.2\%), Parenchymal nodules (7.2\%) and Mediastinal lymphadenopathy (5.7\%). Mild air trapping was observed in three patients. 8 UIP pattern in HRCT thorax was observed in $49.3 \%$ cases of DPLD and $93.2 \%$ in IPF in present study. While it was observed in $42 \%$ in other study. ${ }^{9}$ Ganguly et al observed all cases of IPF ( $n=35,100 \%)$ showed a classical UIP pattern on the HRCT thorax.

\section{REFERENCES}

1. British Thoracic Society. The diagnosis, assessment and treatment of diffuse parenchymal lung disease in adults. Introduction. Thorax 1999;54(1):S1-S14.

2. American Thoracic Society: European Respiratory Society. International multidisciplinary consensus classification of the idiopathic interstitial pneumonias. Am J Respir Crit Care Med 2002;165(2):277-304.

3. Abul A, Onadeko BO, Khadadah ME, et al. Clinical patterns of diffuse parenchymal lung disease in Kuwait: a prospective study. Med Princ Pract 2004;13(2):78-83.

4. Gagia AK, Patel AS, Bhagat GR, et al. Spirometry and X-Ray findings in cases of interstitial lung diseases. National Journal of Community Medicine 2012;3:700-2.

5. Jindal SK, Malik SK, Deodhar SD, et al. Fibrosing alveolitis: a report of 61 cases seen over the past five years. Indian J Chest Dis Allied Sci 1979;21(4):174-9.

6. Verma SK, Prasad R, Chaudhary AS, et al. A study of diffuse parenchymal lung disease. Pulmon 2008;10(3):94-7.

7. Sen T, Udwadia ZF. Retrospective study of interstitial lung disease in a tertiary care centre in India. Indian J Chest Dis Allied Sci 2010;52(4):207-11.

8. Maheshwari U, Gupta D, Aggarwal AN, et al. Spectrum and diagnosis of idiopathic pulmonary fibrosis. Indian J Chest Dis Allied Sci 2004;46(1):23-6.

9. Shafeeq MK, Anithakumari K, Fathahudeen A, et al. Aetiology and clinic-radiological profile of interstitial lung disease in a tertiary care centre. Pulmon 2011;13:12-5.

10. Kumar R, Gupta N, Goel N. Spectrum of interstitial lung disease at a tertiary care centre in India. Pneumonol Alergol Pol 2014;82(3):218-26.

11. Mahashur AA, Dave KM, Kinare SG, et al. Diffuse fibrosing alveolitis in Indian experience. Lung India 1983;1(5):1719.

12. Coultas DB, Zumwalt RE, Black WC. The epidemiology of interstitial lung diseases. American Journal of Respiratory and Critical Care Medicine 1994;150(4):967-72.

13. Alfred P Fishman. Fishman's pulmonary diseases and disorders 4th edition. McGraw-Hill Professional, 2008;1:1105-7.

14. Kundu S, Mitra S, Ganguly J, et al. Spectrum of diffuse parenchymal lung diseases with special reference to idiopathic pulmonary fibrosis and connective tissue disease: an eastern India experience. Lung India 2014;31(4):354-60.

15. Turner M, Burrows B, Johnson A. Cryptogenic fibrosing alveolitis-clinical features and their influence on survival Thorax 1980;35(3):171-80. 
16. Subhash HS, Ashwin I, Solomon SK, et al. A comparative study on idiopathic pulmonary fibrosis and secondary diffuse parenchymal lung disease. Indian J Med Sci 2004;58(5):185-90.

17. Raghu G. Interstitial lung disease: a diagnostic approach. Are CT scan and lung biopsy indicated in every patient? Am J Respir Crit Care Med 1995;151(3 Pt 1):909-14.

18. Savarino E, Carbone R, Marabotto E, et al. Gastrooesophageal reflux and gastric aspiration in idiopathic pulmonary fibrosis patients. Eur Respir J 2013;42(5):1322-31.

19. Schwartz DA, Merchant RK, Helmers RA, et al. The influence of cigarette smoking on lung function in patients with idiopathic pulmonary fibrosis. Am Rev Respir Dis 1991;144(3):504-6.

20. Costabel U, Hunninghake GW. Statement on sarcoidosis: joint statement of ATS, ERS and the world association of sarcoidosis and other granulomatous disorder (WASGO). Eur Respir J 1999;14(4):735-7.

21. Rybicki BA, Major M, Popovich JJ, et al. Racial differences in sarcoidosis incidence: a 5-year study in a health maintenance organization. Am J Epidemiol 1997;145(3):234-41.
22. Vestbo J, Hurd SS, Agustí AG, et al. Global strategy for the diagnosis, management, and prevention of chronic obstructive pulmonary disease: GOLD executive summary. Am J Respir Crit Care Med 2013;187(4):347-65.

23. Andersen CU, Mellemkjaer S, Hilberg O, et al. Pulmonary hypertension in interstitial lung disease: prevalence, prognosis and $6 \mathrm{~min}$ walk test. Respir Med 2012;106(6):875-82.

24. Caminati A, Cassandro R, Harari S. Pulmonary hypertension in chronic interstitial lung diseases. European Respiratory Review 2013;129:292-301.

25. Hamada K, Nagai S, Tanaka S, et al. Significance of pulmonary arterial pressure and diffusion capacity of the lung as prognosticator in patients with idiopathic pulmonary fibrosis. Chest 2007;131(3):650-6.

26. Abeles AM, Abeles M. The clinical utility of a positive antinuclear antibody test result. Am J Med 2013;126(4):342-8.

27. Weinberg I, Vasiliev L, Gotsman I. Anti-dsDNA antibodies in sarcoidosis. Semin Arthritis Rheum 2000;29(5):328-31.

28. Fischer A, West SG, Swigris JJ, et al. Connective tissue disease-associated interstitial lung disease: a call for clarification. Chest 2010;138(2):251-6. 